\title{
Superior Tactile Abilities in the Blind: Is Blindness Required?
}

\author{
Patrice Voss \\ Montreal Neurological Institute, McGill University, Montréal, Québec H3A 2B4, Canada, and International Laboratory for Brain, Music and Sound \\ Research, Montréal, Québec H3C 3J7, Canada \\ Review of Wong et al.
}

How blindness affects a person's other senses has been questioned for some time. One of the primary debates revolved around whether blind individuals would be handicapped beyond the visual realm, given vision's roles in the construction of spatial concepts and for spatial calibration of other senses, or if on the other hand, these functions would be taken over by heightened abilities in the remaining senses. The latter is suggested by numerous anecdotal stories about blind individuals' prowess with auditory and tactile senses, as well as by studies that show enhanced abilities in the blind for both the auditory (Collignon et al., 2009) and tactile (Sathian and Stilla, 2010) domains. These studies have led researchers to explore the driving force underlying these improved abilities. Some of this improvement is likely mediated by massive cross-modal plasticity: areas of the occipital cortex in blind individuals become highly responsive to nonvisual input. Superior behavioral abilities might require the extra cerebral resources allotted to auditory and tactile processing following blindness. On the other hand, blind individuals benefit from extensive training in the auditory and tactile modalities, and this additional experience

Received May 26, 2011; revised June 27, 2011; accepted June 28, 2011.

I thank R. Zatorre and K. Tadros for comments on a previous draft of this manuscript.

Correspondence should be addressed to Patrice Voss, Cognitive Neuroscience Unit, Montreal Neurological Institute, McGill University, 3801 University, Montréal, Québec H3A 2B4, Canada. E-mail: patrice.voss@mcgill.ca.

DOI:10.1523/JNEUROSCI.2624-11.2011

Copyright $\odot 2011$ the authors $\quad 0270-6474 / 11 / 3111745-03 \$ 15.00 / 0$ may be the more important factor underlying superior abilities.

In their recent article published in The Journal of Neuroscience, Wong et al. (2011) examined whether superior ability of blind individuals in a grating orientation task (GOT) was the consequence of visual deprivation alone or whether tactile experience-dependent mechanisms were the key determinant. The authors followed up on a previous study showing that tactile acuity is enhanced in blindness (Goldreich and Kanics, 2003), and they compared the passive tactile spatial acuity on the index, middle, and ring fingers of blind participants with varying levels of Braille expertise to that of sighted participants. The variation in Braille expertise allowed them to assess the effect of tactile training on performance on the GOT. As an additional control condition, acuity was also assessed on the lips, where blind and sighted individuals are more likely to have similar tactile experience compared to fingers.

Several results obtained by Wong et al. (2011) highlight the importance of tactile experience in the development of heightened tactile spatial acuity observed in blind individuals (Goldreich and Kanics, 2003). Blind participants had smaller tactile spatial thresholds on their fingertips than did their agematched sighted counterparts. In contrast, no difference was found between the groups regarding the lips' spatial tactile acuity, strongly suggesting that the lower threshold observed for the fingertips of blind individuals was primar- ily driven by experience-dependent mechanisms in the tactile modality. This claim is further supported by the finding that blind proficient Braille readers, compared to blind nonreaders, had lower GOT thresholds on the reading/dominant hand, but not on the opposite hand. Furthermore, correlational analyses showed that the tactile spatial acuity threshold on the reading finger of blind Braille readers was significantly correlated with the weekly reading time of each subject. As a whole, these robust findings emphasize the impact that prolonged tactile experience can have in shaping the tactile spatial resolution of the trained body parts.

The findings of Wong et al. (2011), along with those of previous research, raise interesting and important questions. Notably, is blindness a necessary prerequisite for the development of heightened sensory abilities? Indeed, it is well established that training in itself leads to improved performance, regardless of the task or the modality at hand. For instance, prior studies have shown that tactile finger acuity can increase in normal sighted individuals through intensive training (Sathian and Zangaladze, 1997; Harris et al., 2001), suggesting that blindness may only act as a facilitator in the development of increased tactile acuity, because it encourages training of the nonvisual senses to compensate for visual deprivation. Moreover, it was shown in the current study (Wong et al., 2011) that the ability of blind nonreaders was not statistically significantly better than sighted subjects, 
even though these blind nonreaders may have used their finger tactile sense for other purposes, such as object exploration and recognition, suggesting that blindness alone is not sufficient for the development of heightened sensory abilities. There is reason to believe, however, that visual deprivation can also directly lead to behavioral enhancements. For example, it has been shown that transient and reversible visual deprivation in sighted individuals can significantly improve tactile acuity, at least on a temporary basis (Kauffman et al., 2002; Facchini and Aglioti, 2003).

Given the strong evidence that both sensory deprivation and extensive training lead to improved sensory abilities, the question then arises as to how they may interact. Does blindness simply motivate tactile training or does the availability of cross-modal plasticity, i.e., the ability of visual cortex in blind individuals to take on nonvisual sensory processing (e.g., Cohen et al., 1997; Voss et al., 2011), provide a unique opportunity for developing heightened sensory abilities? The absence of competitive visual inputs in the occipital cortex of blind individuals may facilitate and accelerate functional and anatomical plasticity resulting from intensive training. Alternatively, a synergy between training and deprivation might also result in a higher performance ceiling for blind individuals in relation to non-visually deprived individuals (Fig. 1). This could be directly tested by comparing sighted individuals that are intensely trained and blindfolded for prolonged periods of time with individuals that were either only trained or only blindfolded-although finding participants willing to be blindfolded over lengthy periods might prove to be difficult. Similarly, one could compare blind individuals with sighted ones before and after intensive training. Such experiments would help determine whether sensory deprivation does indeed raise the performance ceiling or hastens the effects of training. It is also possible that neither of these effects would occur, but a recent study in the auditory domain suggests that this is unlikely. Wan et al. (2010) showed that blind individuals outperformed sighted ones on a range of pitch-based auditory tasks even though musical experience was controlled for, suggesting that visual deprivation interacts with auditory training.

A final point worth noting is the finding that early- and late-onset blind individuals showed comparable tactile spatial
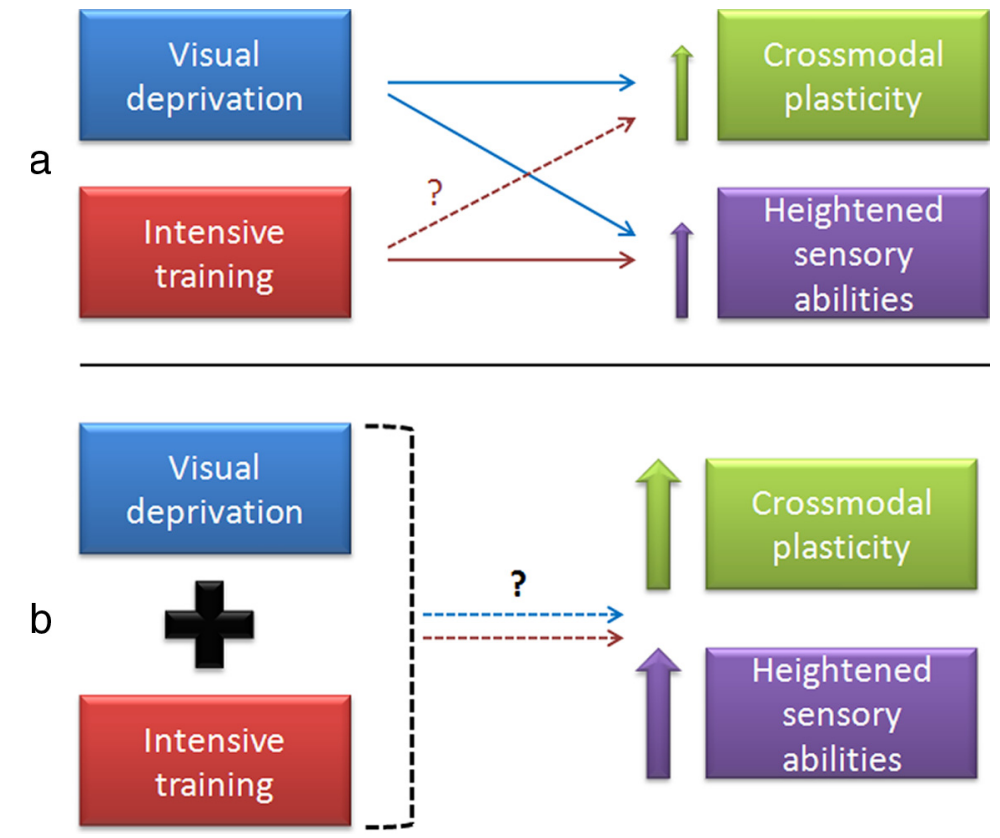

Figure 1. Schematic illustration of the influence of visual deprivation (VD) and intensive training (IT) on the development of heightened sensory abilities and cross-modal plasticity. $\boldsymbol{a}$ illustrates how VD and IT are known to independently increase cross-modal plasticity and nonvisual abilities. The dashed line illustrates the fact there is currently little to no evidence showing that intensive training alone can lead to similar cross-modal changes as observed in VD studies. Rather, intensive training typically leads to intramodal plastic changes. $\boldsymbol{b}$ illustrates a hypothetical synergic relationship between VD and IT leading to even further heightened sensory abilities and cross-modal plasticity relative to that observed under either condition alone.

thresholds (Wong et al., 2011). This finding argues against the importance of the early onset of visual deprivation for the improvement of sensory abilities as well as to benefit from cross-modal recruitment of the deafferented occipital cortex, which is in marked contrast with many results in the literature (Cohen et al., 1999; Sadato et al., 2002). One must then keep in mind that the particular role of training reported by Wong et al. (2011) might be specific to the type of task or the sensory modality used, and may not be generalizable to all superior abilities observed in the blind population. Moreover, the relationship between sensory deprivation and training may qualitatively differ between sensory modalities. Therefore similar work will need to take place with different tasks and modalities, notably in the auditory domain, where behavioral differences between early and late blind subjects have often been reported.

In conclusion, the study by Wong et al. (2011) addresses an important issue in the study of heightened sensory abilities that follow the loss of sight by assessing the role of experience-based learning in relation to the role played by visual deprivation itself. The authors showed the tactile spatial acuity of blind subjects to be superior to that of sighted individuals for their fingers but not for their lips, suggesting that experiencedependent mechanisms drive this improvement as opposed to visual deprivation. This claim is further supported by the finding that finger acuity is greater in proficient Braille readers than nonreaders, and by the fact that finger acuity was shown to positively correlate with weekly reading time in Braille readers. These findings raise significant questions regarding the role that visual deprivation in itself plays in the development of heightened sensory abilities. Addressing these questions would help ascertain the true role of visual deprivation in the development of improved sensory abilities, whether it be necessary for optimal performance via synergistic processes, constitute an alternative to intensive training, or simply act as a facilitatory agent in allowing for more committed training to compensate for the visual loss.

\section{References}

Cohen LG, Celnik P, Pascual-Leone A, Corwell B, Falz L, Dambrosia J, Honda M, Sadato N, Gerloff C, Catalá MD, Hallett M (1997) Functional relevance of cross-modal structural changes in blind humans. Nature 389:180-183.

Cohen LG, Weeks RA, Sadato N, Celnik P, Ishii K, 
Hallett M (1999) Period of susceptibility for cross-modal structural changes in the blind. Ann Neurol 45:451-460.

Collignon O, Voss P, Lassonde M, Lepore F (2009) Cross-modal plasticity for the spatial processing of sounds in visually deprived subjects. Exp Brain Res 192:343-358.

Facchini S, Aglioti SM (2003) Short-term light deprivation increases tactile spatial acuity in humans. Neurology 60:1998-1999.

Goldreich D, Kanics IM (2003) Tactile acuity is enhanced in blindness. J Neurosci 23: $3439-3445$.

Harris JA, Harris IM, Diamond ME (2001) The topography of tactile learning in humans. J Neurosci 21:1056-1061.

Kauffman T, Théoret H, Pascual-Leone A (2002) Braille character discrimination in blindfolded human subjects. Neuroreport 13:571-574.

Sadato N, Okada T, Honda M, Yonekura Y (2002) Critical period for cross-modal plasticity in blind humans: a functional MRI study. Neuroimage 16:389-400.

Sathian K, Stilla R (2010) Cross-modal plasticity of tactile perception in blindness. Restor Neurol Neurosci 28:271-281.

Sathian K, Zangaladze A (1997) Tactile learning is task specific but transfers between fingers. Percept Psychophys 59:119-128.

Voss P, Lepore F, Gougoux F, Zatorre RJ (2011) Relevance of spectral cues for auditory spatial processing in the occipital cortex of the blind. Front Psychol 2:48.

Wan CY, Wood AG, Reutens DC, Wilson SJ (2010) Early but not late-blindness leads to enhanced auditory perception. Neuropsychologia 48:344-348.

Wong M, Gnanakumaran V, Goldreich D (2011) Tactile spatial acuity enhancement in blindness: evidence for experience-dependent mechanisms. J Neurosci 31:7028-7037. 